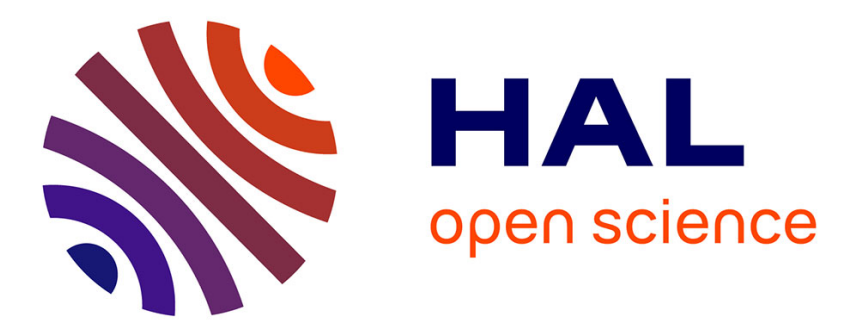

\title{
Le délai diagnostique de la spondyloarthrite axiale : une étude transversale sur 432 patients
}

\author{
Vanina Masson Behar, Maxime Dougados, Adrien Etcheto, Sarah Kreis, \\ Stéphanie Fabre, Christophe Hudry, Sabrina Dadoun, Christopher Rein, \\ Edouard Pertuiset, Bruno Fautrel, et al.
}

\section{To cite this version:}

Vanina Masson Behar, Maxime Dougados, Adrien Etcheto, Sarah Kreis, Stéphanie Fabre, et al.. Le délai diagnostique de la spondyloarthrite axiale : une étude transversale sur 432 patients. Revue du Rhumatisme, 2017, 10.1016/j.rhum.2017.07.035 . hal-01592544

\section{HAL Id: hal-01592544 https://hal.sorbonne-universite.fr/hal-01592544}

Submitted on 25 Sep 2017

HAL is a multi-disciplinary open access archive for the deposit and dissemination of scientific research documents, whether they are published or not. The documents may come from teaching and research institutions in France or abroad, or from public or private research centers.
L'archive ouverte pluridisciplinaire HAL, est destinée au dépôt et à la diffusion de documents scientifiques de niveau recherche, publiés ou non, émanant des établissements d'enseignement et de recherche français ou étrangers, des laboratoires publics ou privés. 
Délai diagnostique de la spondylarthrite axiale : une étude transversale portant sur $\mathbf{4 3 2}$ patients

Vanina Masson Behar ${ }^{1,2,3}$, Maxime Dougados ${ }^{3}$, Adrien Etcheto ${ }^{3}$, Sarah Kreis ${ }^{1,2}$, Stéphanie Fabre ${ }^{1,2,4}$, Christophe Hudry ${ }^{3}$, Sabrina Dadoun ${ }^{1,2}$, Christopher Rein ${ }^{1,2}$, Edouard Pertuiset ${ }^{4}$, Bruno Fautrel, ${ }^{1,2}$ and Laure Gossec ${ }^{1,2}$

1Sorbonne Universités, UPMC Univ Paris 06, GRC-08, Institut Pierre Louis d'Epidémiologie et de Santé Publique, Paris, France;

${ }^{2}$ Hôpital Pitie-Salpétrière, AP-HP, Service de Rhumatologie, Paris, France

${ }^{3}$ Université Paris Descartes, AP-HP, Rhumatologie, Hôpital Cochin, Paris France

${ }^{4}$ Hôpital René Dubos, Pontoise France

\section{AUTEUR CORRESPONDANT}

Laure Gossec, Service de rhumatologie, Hôpital Pitié-Salpétrière, 47-83, Bd de l'Hôpital, 75013 Paris France

laure.gossec@aphp.fr

Tel. $+33-142178421$ 


\section{Abstract}

Objectifs. Le délai diagnostique des formes axiales de spondylarthrite (axSpA) est estimé à plus de sept ans mais semble avoir été raccourci récemment. L'objectif était de quantifier le délai diagnostique chez les patients atteints de axSpA en France et d'en identifier les facteurs associés.

Méthodes. Deux études observationnelles transversales ont inclus consécutivement des patients atteints de axSpA (selon les critères ASAS et l'évaluation du rhumatologue). Le délai diagnostique a été défini par l'intervalle entre la date d'apparition des premiers symptômes et celle du diagnostic. Les facteurs prédictifs potentiels du délai diagnostique analysés par régression linéaire multiple étaient les facteurs démographiques, l'antigène HLA B27, l'année civile du diagnostic, la présentation clinique et la sacro-iliite radiographique ou à l'IRM.

Résultats. Au total, 432 patients ont été analysés : l'âge moyen au diagnostic était de 34,2 (Ecart-type 12,5) ans et la durée moyenne de la maladie au moment de l'évaluation de 11,4 $(10,4)$ ans. Au total, $66,7 \%$ des patients étaient HLA B27 positifs et $70,2 \%$ des sujets présentaient une sacro-iliite à la radiographie. Le délai diagnostique moyen s'élevait à 4,9 $(6,3)$ ans, avec une valeur médiane de 2,0 ans (écart interquartile, 1-7; étendue : 0-43). En analyse multivariable, les facteurs associés de manière indépendante à un délai diagnostique plus long étaient : un âge plus élevé au diagnostic (bêta $=0,13 ; p<0,001$ ), la présence plus rare d'une arthrite périphérique ou d'une dactylite (bêta $=-1,69 \mathrm{p}=0,005$ ) et des douleurs enthésitiques plus fréquentes (bêta $=1,46 ; p=0,015)$.

Conclusion. Le délai diagnostique médian était de 2 ans ce qui montre dans la majorité des cas un délai plus court que celui jusqu'alors rapporté. Une présentation clinique plus "typique" de la SpA était associée à un délai diagnostique plus court, tandis que la sacro-iliite radiographique et la positivité de HLA B27 n'étaient pas des facteurs associés à ce délai.

Mots clé: spondylarthrite, délai, diagnostic 


\section{Introduction}

La spondylarthrite axiale (axSpA) est une maladie inflammatoire chronique caractérisée par la présence de multiples manifestations au niveau du rachis ou des articulations périphériques mais également de diverses atteintes extra-articulaires. Le diagnostic de axSpA est souvent difficile à établir, avec un délai moyen allant de cinq à onze ans entre le début des symptômes et le diagnostic comme confirmé par de récentes études [1-6]. Dernièrement, une large étude du registre Danois DANBIO a toutefois montré une réduction de la durée moyenne entre l'apparition des premiers symptômes et l'établissement du diagnostic de SpA axiale qui est passée de 5,5 ans en 2000 à 3-4 mois en 2011 [7]. Un diagnostic précoce est indispensable pour permettre d'instaurer le premier traitement, améliorer les stratégies de coping et diminuer l'anxiété naturelle des patients [3,8]. Le pronostic des patients souffrant de polyarthrite rhumatoïde s'améliore lorsque le diagnostic est posé au plus tôt après le début des symptômes [9]. II n'y a pas, à l'heure actuelle, de données suffisantes à ce sujet concernant la SpA, le principe d'une consultation rapide et d'un diagnostic effectué précocement est toutefois recommandé dans la SpA axiale $[10,11,12,13]$. De plus, les patients atteints de axSpA sont parfois soumis à de nombreuses explorations avant le diagnostic qui peuvent s'avérer inutiles voire invasives.

Dans un grand nombre de cas, la présence d'un tableau clinique incomplet tel qu'une lombalgie isolée ou des symptômes extra-articulaires mais aussi l'absence de tests diagnostiques pathognomoniques cliniques ou biologiques et de critères diagnostiques validés sont autant de facteurs susceptibles d'expliquer le retard diagnostique dans la SpA axiale $[2,4,14]$. Les facteurs démographiques semblent aussi jouer un rôle : on observe un délai diagnostique plus long chez les femmes que chez les hommes ; le niveau d'éducation pourrait également avoir un impact sur ce délai [2,5,14]. Par ailleurs, le délai diagnostique apparaît plus court chez les patients porteurs de l'antigène HLA B27 [1]. L'apparition souvent retardée d'une sacro-iliite radiographique, essentielle au diagnostic de SpA axiale, contribue elle aussi au retard diagnostique [15,16]. En effet, la progression radiographique est lente avec la présence d'une sacro-iliite radiographique selon les critères de New-York modifiés chez seulement $70 \%$ des patients atteints de SpA axiale, 5 ans après l'apparition des premiers symptômes [17]. La reconnaissance de la SpA axiale non radiographique à partir des critères ASAS (Assessment of Spondyloarthritis international Society) constitue une récente avancée dans le domaine de la $\mathrm{SpA}$ puisque ces critères de classification comprennent la présence d'une sacro-iliite à l'IRM [16,18-20]. La présente étude avait pour objectifs d'analyser les facteurs associés au délai diagnostique et d'explorer dans un sous-groupe de patients les procédures et investigations réalisées durant la période allant de l'apparition des premiers symptômes jusqu'au diagnostic. 


\section{Méthodes}

\subsection{Design de l'étude}

Deux études observationnelles multicentriques ont été menées (respectivement en 2009 et 2013) dans deux centres de soins tertiaires, un centre de soins secondaires et un cabinet privé à Paris, comme précédemment publié [21,22]. II s'agit d'études à caractère rétrolectif (recueil prospectif de données rétrospectives). Les études ont été réalisées dans le respect des principes éthiques applicables en France, le consentement oral a été obtenu de tous les patients et le comité d'éthique de l'Hôpital de la Pitié Salpétrière a donné son accord pour la réalisation de la seconde étude [22].

\subsection{Patients}

Les patients étaient atteints de SpA axiale selon le diagnostic du rhumatologue et les critères de classification de l'ASAS pour la SpA axiale [16,18-20]. Tous les patients étaient âgées d'au moins 18 ans, parlaient le français et étaient capables et désireux de répondre au questionnaire. Un échantillon aléatoire des patients qui répondaient à ces critères a été contacté dans chaque centre.

Dans la première étude, les patients ont été vus à l'occasion d'une hospitalisation ou d'une consultation en soins externes à l'Hôpital Cochin de Paris pendant 6 mois durant 2009 et les données ont été recueillies lors d'entretiens en face-à-face puis complétées par les dossiers médicaux [21]. Dans la seconde étude, des patients issus d'autres centres incluant l'Hôpital de la Pitié Salpétrière à Paris, ont été contactés par mail en 2013 et invités à remplir un autoquestionnaire. Les données médicales ont été obtenues en ayant recours aux dossiers médicaux [22].

Seuls les patients dont la date d'apparition des symptômes et celle du diagnostic étaient renseignées ont été inclus dans l'analyse ; les patients dont la date d'apparition de la maladie était antérieure à celle des premiers symptômes, ont été exclus (47 patients).

\subsection{Délai diagnostique}

Le délai diagnostique a été défini comme étant la période entre l'apparition des premiers symptômes (de type axial ou non-axial) et la date du diagnostic, telle que définie par le patient et confirmée par le dossier médical. 


\subsection{Les facteurs associés au délai diagnostique}

Les facteurs démographiques comprenant le sexe, l'âge au moment du diagnostic, le niveau d'éducation ( $\leq$ fin des études secondaires, > études secondaires), la situation professionnelle (emploi rémunéré ou pas), la situation socio-économique (fonctionnaire ou autres catégories), l'année civile du diagnostic et la présence du HLA B27 ont été enregistrés. Les données relatives à la présentation clinique au cours de la maladie incluant la présence d'une arthrite périphérique, une dactylite ou des douleurs enthésitiques, les manifestations extra-articulaires (uvéite, maladie inflammatoire intestinale, psoriasis) et la réponse aux anti-inflammatoires non-stéroïdiens (AINS) ont également été collectées. La sacro-iliite a été définie à partir des lectures locales des clichés radiographiques (selon les critères de New-York modifiés) ou de I'IRM réalisées à tout moment au cours de la maladie et comme disponible dans le dossier médical.

\subsection{Description des évènements avant le diagnostic}

Les évènements survenus avant le diagnostic ont été analysés dans la première étude, chez 265 patients [21]. Le nombre de médecins consultés avant le diagnostic et leur spécialisation, les procédures inutiles/invasives avant le diagnostic (saccoradiculographie et autres explorations telles que la coloscopie, le myélogramme, la biopsie synoviale, la scintigraphie osseuse, l'arthroscopie ou la synovectomie) et les traitements instaurés avant le diagnostic qui présentent une éventuelle toxicité comme les immunosuppresseurs, les corticoïdes oraux, les infiltrations articulaires ou rachidiennes et la chirurgie ont été recueillis.

\subsection{Analyses Statistiques}

Les facteurs associés au délai diagnostique ont été analysés par régression linéaire uni et multivariée. L'analyse univariée incluait les variables qui semblaient intéressantes à la lumière de la littérature et disponibles dans les deux cohortes. Toutes les variables de valeur $p<0,20$ en analyse univariée ont ensuite été intégrées à l'analyse multivariée selon une méthode de sélection ascendante à l'aide du logiciel SAS (Statistical Analysis System) version 9.4. Aucune imputation n'a été effectuée pour les données manquantes.

\section{Résultats}

\subsection{Patients}

Parmi les 479 participants aux études, 432 ont été analysés (Tableau 1) : l'âge moyen au moment du diagnostic était de 34,2 (écart type, ET, 12,5) ans et l'âge moyen à l'apparition des 
premiers symptômes de 29,3 (ET 12,2) ans. Au total, 288/383 (75,1\% des patients ayant des données disponibles) étaient HLA B27 positifs, 278/396 (70,2\%) avaient une sacro-iliite radiologique après lecture des clichés radiographiques par un investigateur local et 138/251 $(55,0 \%)$ avaient une sacro-iliite à I'IRM. L'année 2000 était l'année moyenne au cours de laquelle était posé le diagnostic (ET 10). Au moment de l'évaluation, la durée moyenne de la maladie était de 11,4 ans (ET 10,4), 271 (62,7\%) patients étaient sous traitement anti-TNF et $329(77,6 \%)$ avaient un emploi rémunéré.

\subsection{Délai diagnostique}

Le délai diagnostique moyen était de 4,9 (ET 6,3) ans, avec une médiane de 2,0 ans (écart interquartile, 1-7; étendue : 0-43). (Figure 1) Les deux cohortes ont obtenu des scores identiques (données non communiquées).

\subsection{Facteurs associés au délai diagnostique}

En analyse univariée, le sexe féminin (bêta=-0,91 $p=0,14$ ), un âge plus avancé au moment du diagnostic (bêta $=0,15 ; p<0,001$ ), un niveau d'éducation inférieur ( $\leq$ fin des études secondaires, bêta $=-1,66 ; p=0,034)$, une année civile plus élevée au moment du diagnostic (bêta $=0,079, p=0,005$ ), l'absence d'emploi rémunéré (bêta $=-1,75, p=0,005$ ), la survenue moins fréquente d'une arthrite périphérique ou d'une dactylite (bêta=-1,92; $p=0,016$ ), et des douleurs enthésitiques plus fréquentes (bêta $=1,10 ; p=0,074)$ étaient des facteurs associés à un diagnostic tardif de manière suffisamment significative pour être intégrés dans l'analyse multivariée (Tableau 2). La positivité de HLA B27, le statut socio-économique, les manifestations extra-articulaires de la SpA, la réponse aux AINS et la sacro-iliite (radiographique ou IRM) n'étaient pas des facteurs associés au délai diagnostique (données non-communiquées). En analyse multivariée (412 patients avec données disponibles), les facteurs associés de manière indépendante à un délai diagnostique plus long étaient : un âge plus élevé au diagnostic, la présence plus rare d'une arthrite périphérique ou d'une dactylite et des douleurs enthésitiques plus fréquentes (Tableau 2). Le facteur "année civile du diagnostic" n'était pas considéré comme étant significatif.

\subsection{Description des évènements survenus avant le diagnostic}

Parmi les 265 patients de l'étude de 2009, les explorations invasives ou inutiles réalisées avant le diagnostic ont été étudiées. Les patients avaient consulté en moyenne 2,8 médecins (médiane : 2.0) avant le diagnostic : 69,1\% s'étaient adressés à un rhumatologue et 8,6\% à un chirurgien orthopédiste. Au total, 2 patients $(0,7 \%)$ ont subi une saccoradiculographie et 28 $(10,6 \%)$ d'autres examens invasifs : scintigraphie osseuse $(\mathrm{N}=13)$, coloscopie $(\mathrm{N}=2)$, biopsie 
sternale $(\mathrm{N}=1)$, biopsie synoviale $(\mathrm{N}=7)$, arthroscopie $(\mathrm{N}=3)$, et/ou synovectomie $(\mathrm{N}=2)$. Les traitements administrés durant la période pré-diagnostique incluaient des infiltrations rachidiennes ou articulaires chez 60 (22,6\%) patients, une corticothérapie orale chez 36 $(13,2 \%)$, des immunosuppresseurs chez $14(5,3 \%)$, et une chirurgie chez 5 (1,9\%) : une chirurgie rachidienne chez 3 , une arthrodèse de l'articulation interphalangienne proximale chez 1 et une arthroplastie totale de la hanche chez un.

\section{Discussion}

Le délai diagnostique médian chez ces patients atteints de SpA axiale était de 2 ans, ce qui montre un délai plus court pour la plupart des patients avec SpA axiale comparativement à celui rapporté jusqu'alors. Cependant, quelques patients avaient un délai diagnostique très long qui, pour certains d'entre eux, a conduit à la réalisation de procédures diagnostiques ou thérapeutiques inutiles voire dangereuses. Une présentation clinique plus "typique" de la SpA, en particulier sans enthésite, était associée à un délai diagnostique plus court, une telle association n'étant pas observée avec la positivité de HLA B27 et la sacro-iliite à l'imagerie, ce qui indique que les $\mathrm{SpA}$ axiales non-radiographiques sont clairement reconnues en France.

Cette large étude offre un aperçu intéressant sur le délai diagnostique de la SpA axiale. Parmi les faiblesses de ce travail, on note la présentation clinique et la sacro-iliite radiographique ou IRM qui sont des critères évalués pendant la durée de la maladie mais pas à la date du diagnostic de la $\mathrm{SpA}$. L'idéal serait de recueillir de manière prospective les différentes manifestations de la maladie dans le temps. Le biais de rappel de cette étude est une autre source potentielle d'erreurs : les patients ayant une maladie de plus longue durée ont plus de difficultés à se souvenir de la date d'apparition des symptômes et de celle du diagnostic; c'est pourquoi nous avons exclu 47 patients dont la date de survenue de la maladie était antérieure à la date des premiers symptômes ; les dates ont toutefois été confirmées aussi souvent que possible par les dossiers médicaux. Enfin, nous savons que les résultats de cette étude ne sont pas le reflet exact des délais diagnostiques globaux en France, la plupart des patients analysés étant suivis en milieu hospitalier; cependant, un certain nombre de participants était issu de services de soins secondaires ou de cabinets privés ce qui permet de généraliser davantage les résultats obtenus.

Le délai diagnostique médian dans la présente étude était de 2,0 ans. Les données de la littérature à ce sujet sont hétérogènes mais font généralement état d'un délai diagnostique plus long [2-6]. La récente étude Danoise a retrouvé un délai inférieur à 6 mois au cours de 
l'année précédente [7]. Notre étude ne nous permet pas de comparer l'évolution du délai diagnostique puisqu'il s'agit d'une évaluation transversale de patients atteints de SpA axiale pour lesquels la durée de la maladie était variable et souvent élevée [7]. C'est pourquoi, le délai relativement court observé dans notre étude est surprenant dans la mesure où le diagnostic a été posé aux environs de l'année 2000. La meilleure reconnaissance de la SpA axiale en France à partir des critères d'Amor permet d'expliquer en partie ces résultats.

Dans la présente étude, le HLA B27, le sexe féminin, le niveau d'éducation, la sacro-iliite à I'IRM et l'année du diagnostic n'étaient pas des facteurs associés de manière significative à un délai diagnostique plus élevé même si de tels facteurs ont déjà été retrouvés dans la littérature $[1,2,5,14]$. Cependant, le pourcentage de patients HLA-B27 positifs était conforme à nos prévisions $(75,1 \%)$. En ce qui concerne la présence d'une sacro-iliite à l'IRM, nos résultats ne permettent pas de préciser son rôle dans le délai diagnostique en raison du faible nombre de résultats IRM disponibles, de l'utilisation limitée de cette technique d'imagerie autour de l'année 2000 et parce qu'il s'agissait d'un critère évalué pendant toute la durée de la maladie du patient. Les résultats impressionnants de la récente étude nationale Danoise sont certainement liés à un usage plus large de l'IRM ces dernières années. L'IRM est une technique d'imagerie utile mais parfois difficile d'interprétation [20,23]. Les critères de classification de l'ASAS, qui définissent les formes axiales non-radiographiques de la SpA permettent vraisemblablement de réduire le délai diagnostique. Dans la présente étude, l'absence de lien entre la sacro-iliite radiographique et le délai diagnostique indique que la SpA axiale non-radiographique est un concept bien reconnu en France. Ce constat est sans doute la conséquence de l'utilisation répandue en pratique clinique en France des critères d'Amor qui, bien avant les critères ASAS, reconnaissaient certaines formes de SpA sans sacro-iliite radiographique $[19,24,25]$. D'un autre côté, il existe un risque de sur-diagnostic de la SpA axiale, et le suivi à long terme des patients atteints d'une forme non-radiographique a donc tout son intérêt.

Dans la présente étude, l'âge plus élevé et la présentation clinique - en particulier les douleurs enthésitiques et l'absence d'arthrite périphérique - constituaient des facteurs prédictifs d'un délai diagnostique plus long. La détection de la SpA axiale, lorsqu'elle survient avant l'âge de 30 ans, semble plus évidente en raison du risque moins élevé d'erreur de classification en présence d'une douleur du dos non-spécifique et d'une hernie discale dégénérative $[2,4,26,27]$. Par ailleurs, les douleurs enthésitiques ne sont pas considérées comme étant caractéristiques puisque des symptômes similaires peuvent être retrouvés chez les patients en surpoids, les sportifs ou les sujets souffrant de fibromyalgie, ce qui pourrait expliquer leur lien avec le délai diagnostique [28]. Inversement, et comme indiqué ici, l'arthrite périphérique peut être considérée comme un "signal d'alarme" chez les jeunes patients souffrant de 
douleurs lombaires [29,30]. Dans un sous-groupe de patients de cette étude, les évènements précédant le diagnostic ont été explorés. Les patients avaient consulté en moyenne 2,8 médecins, ce chiffre étant le reflet d'une certaine errance diagnostique [2,4]. Une proportion significative de patients avait fait l'objet d'examens invasifs ou injustifiés mais également de traitements potentiellement dangereux incluant la chirurgie, ces données confirmant celles d'une précédente étude selon laquelle $6,6 \%$ des patients atteints d'une SpA de forme axiale avaient subi une intervention chirurgicale pour hernie discale lombaire avant le diagnostic [2].

D'après les recommandations formulées au sujet de la SpA, le délai diagnostique est un enjeu majeur qui nécessite d'être considéré avec la plus grande attention et des stratégies de prise en charge précoce ont été instaurées [7,31-35]. Les systèmes de santé et d'accès aux soins diffèrent d'un pays à l'autre pouvant alors influencer le délai diagnostique [36]. La France compte un nombre important de rhumatologues et son système d'assurance maladie permet d'accéder directement à un spécialiste. Pour conclure, en dépit du délai diagnostique plus court observé dans cette étude comparativement à celui jusqu'alors publié, d'autres études seront nécessaires pour évaluer la place de l'IRM dans la démarche diagnostique ainsi que les éventuelles conséquences d'un diagnostic plus tardif mais également d'un sur-diagnostic de la SpA axiale.

\section{AUTEURS ET CONTRIBUTEURS}

Tous les auteurs :

- Ont contribué de manière substantielle à l'élaboration de l'étude, au recueil, à l'analyse ou à l'interprétation des données de l'étude; ET

- $\quad$ Ont participé à la préparation de l'étude ou à la révision critique du contenu intellectuel important; ET

- $\quad$ Ont approuvé la version finale à publier; ET

- Engagent leur responsabilité pour tous les aspects de ce travail et garantissent l'exactitude et l'intégrité des informations publiées.

Les auteurs qui ont accepté d'engager leur entière responsabilité pour ce travail et/ou la réalisation de l'étude, ont eu accès aux données et ont supervisé la décision de publication de cet article sont Vanina Masson Behar et Laure Gossec. 


\section{Déclaration d'intérêts}

Les auteurs ne déclarent aucun conflit d'intérêts en lien avec cette étude. 


\section{Bibliographie}

[1] Chung HY, Machado P, van der Heijde D, D'Agostino MA, Dougados M. HLA-B27 positive patients differ from HLA-B27 negative patients in clinical presentation and imaging: results from the DESIR cohort of patients with recent onset axial spondyloarthritis. Ann Rheum Dis. $2011 ; 70(11): 1930-6$

[2] Gerdan V, Akar S, Solmaz D, Pehlivan Y, Onat AM, Kisacik B et al. Initial diagnosis of lumbar disc herniation is associated with a delay in diagnosis of ankylosing spondylitis. $\mathrm{J}$ Rheumatol. 2012;39(10):1996-9

[3] Salvadorini G, Bandinelli F, Delle Sedie A, Riente L, Candelieri A, Generini S et al. Ankylosing spondylitis: how diagnostic and therapeutic delay have changed over the last six decades. Clin Exp Rheumatol. 2012;30(4):561-5

[4] Aggarwal R, Malaviya AN. Diagnosis delay in patients with ankylosing spondylitis: factors and outcomes-an Indian perspective. Clin Rheumatol. 2009;28(3):327-31

[5] Feldtkeller E, Bruckel J, Khan MA. Scientific contributions of ankylosing spondylitis patient advocacy groups. Curr Opin Rheumatol. 2000;12(4):239-47

[6] Feldtkeller E, Erlendsson J. Definition of disease duration in ankylosing spondylitis. Rheumatol Int. 2008;28(7):693-6

[7] Sørensen J, Hetland ML; all departments of rheumatology in Denmark. Diagnostic delay in patients with rheumatoid arthritis, psoriatic arthritis and ankylosing spondylitis: results from the Danish nationwide DANBIO registry Ann Rheum Dis. 2015 ;74(3):e12

[8] Seo MR, Baek HL, Yoon HH, Ryu HJ, Choi HJ, Baek HJ et al. Delayed diagnosis is linked to worse outcomes and unfavourable treatment responses in patients with axial spondyloarthritis. Clin Rheumatol. 2015 Aug;34(8):1397-405

[9] Smolen JS, Breedveld FC, Burmester GR, Bykerk V, Dougados M, Emery P et al. Treating rheumatoid arthritis to target: 2014 update of the recommendations of an international task force. Ann Rheum Dis. 2016;75(1):3-15.

[10] Smolen JS, Braun J, Dougados M, Emery P, Fitzgerald O, Helliwell P et al. Treating spondyloarthritis, including ankylosing spondylitis and psoriatic arthritis, to target: recommendations of an international task force. Ann Rheum Dis. 2014;73(1):6-16

[11] Sieper J, Rudwaleit M. Early referral recommendations for ankylosing spondylitis (including pre-radiographic and radiographic forms) in primary care. Ann Rheum Dis. 2005;64(5):659-63

[12] Brandt HC, Spiller I, Song IH, Vahldiek JL, Rudwaleit M, Sieper J. Performance of referral recommendations in patients with chronic back pain and suspected axial spondyloarthritis. Ann Rheum Dis. 2007;66(11):1479-84

[13] Robinson PC, Brown MA. The window of opportunity: a relevant concept for axial 
spondyloarthritis. Arthritis Res Ther. 2014;16(3):109

[14] Roussou E, Sultana S. Spondyloarthritis in women: differences in disease onset, clinical presentation, and Bath Ankylosing Spondylitis Disease Activity and Functional indices (BASDAI and BASFI) between men and women with spondyloarthritides. Clin Rheumatol. 2011;30(1):121-7

[15] Van der Linden S, Valkenburg HA, Cats A. Evaluation of diagnostic criteria for ankylosing spondylitis. A proposal for modification of the New York criteria. Arthritis Rheum. 1984;27(4):361-8

[16] Navallas M, Ares J, Beltrán B, Lisbona MP, Maymó J, Solano ARadiographics. Sacroiliitis associated with axial spondyloarthropathy: new concepts and latest trends. 2013;33(4):93356

[17]. Mau W, Zeidler H, Mau R, Majewski A, Freyschmidt J, Stangel W et al. Clinical features and prognosis of patients with possible ankylosing spondylitis. Results of a 10-year followup. J Rheumatol. $1988 ; 15(7): 1109-14$

[18] Sieper J, van der Heijde D. Review: Nonradiographic axial spondyloarthritis: new definition of an old disease? Arthritis Rheum. 2013;65(3):543-51

[19] Rudwaleit M, van der Heijde D, Landewé R, Listing J, Akkoc N, Brandt J et al. The development of Assessment of SpondyloArthritis international Society classification criteria for axial spondyloarthritis (part II): validation and final selection. Ann Rheum Dis 2009;68:777-83 [20] Rudwaleit M, Jurik AG, Hermann KG, Landewé R, van der Heijde D, Baraliakos X et al. Defining active sacroiliitis on magnetic resonance imaging (MRI) for classification of axial spondyloarthritis: a consensual approach by the ASAS/OMERACT MRI group. Ann Rheum Dis. $2009 ; 68(10): 1520-7$

[21] Fabreguet I, Koumakis E, Burki V, Durnez A, Elhai M, Meyer M et al. Assessment of work instability in spondyloarthritis: a cross-sectional study using the ankylosing spondylitis work instability scale. Rheumatology (Oxford). 2012;51(2):333-7

[22] Kreis S, Molto A, Bailly F, Dadoun S, Fabre S, Rein C et al. Relationship between optimism and quality of life in patients with two chronic rheumatic diseases: axial spondyloarthritis and chronic low back pain: a cross sectional study of 288 patients. Health Qual Life Outcomes. 2015;13:78

[23] Sieper J, Rudwaleit M, Baraliakos X, Brandt J, Braun J, Burgos-Vargas R et al. The Assessment of SpondyloArthritis international Society (ASAS) handbook: a guide to assess spondyloarthritis, Ann Rheum Dis. 2009;68 Suppl 2:ii1-44

[24] Dougados $M$, Etcheto $A$, Molto $A$, Alonso $S$, Bouvet $S$, Daurès JP et al. Clinical presentation of patients suffering from recent onset chronic inflammatory back pain suggestive of spondyloarthritis: The DESIR cohort. Joint Bone Spine. 2015 ;82(5):345-51

[25] Amor B, Dougados M, Mijiyawa M. Criteria of the classification of spondylarthropathies. 
Rev Rhum Mal Osteoartic. 1990;57(2):85-9

[26] Jadon DR, Ramanan AV, Sengupta R. Juvenile versus adult-onset ankylosing spondylitis clinical, radiographic, and social outcomes. a systematic review. J Rheumatol. 2013;40(11):1797-805

[27] Guan M, Wang J, Zhu Z, Xiao J, Zhao L, Li Z et al. Comparison in clinical features and life impact between juvenile-onset and adult-onset ankylosing spondylitis. Turk $\mathrm{J}$ Med Sci. 2014;44(4):601-5

[28] Eder L, Jayakar J, Thavaneswaran A, Haddad A, Chandran V, Salonen D et al. Is the MAdrid Sonographic Enthesitis Index useful for differentiating psoriatic arthritis from psoriasis alone and healthy controls? J Rheumatol. 2014;41(3):466-72

[29] Poddubnyy D, van Tubergen A, Landewé R, Sieper J, van der Heijde D, Assessment of SpondyloArthritis international Society (ASAS). Development of an ASAS-endorsed recommendation for the early referral of patients with a suspicion of axial spondyloarthritis. Ann Rheum Dis. 2015;74(8):1483-7 [30] van Hoeven L, Luime J, Han H, Vergouwe Y, Weel A. Identifying axial spondyloarthritis in Dutch primary care patients, ages 20-45 years, with chronic low back pain. Arthritis Care Res (Hoboken). 2014 ;66(3):446-53

[31] Villeneuve E, Nam JL, Bell MJ, Deighton CM, Felson DT, Hazes JM et al. A systematic literature review of strategies promoting early referral and reducing delays in the diagnosis and management of inflammatory arthritis. Postgrad Med J. 2013 ;89(1050):231-40

[32] Combe B, Landewe R, Lukas C, Bolosiu HD, Breedveld F, Dougados M et al, EULAR recommendations for the management of early arthritis: report of a task force of the European Standing Committee for International Clinical Studies Including Therapeutics (ESCISIT). Ann Rheum Dis. 2007 ;66(1):34-45

[33] Muñoz-Fernández S, Carmona L, Collantes E, Mulero J, García-Yébenes MJ, de Miguel E et al; Esperanza Group. A model for the development and implementation of a national plan for the optimal management of early spondyloarthritis: the Esperanza Program. Ann Rheum Dis. 2011;70(5):827-30

[34] Rudwaleit M, Sieper J. Referral strategies for early diagnosis of axial spondyloarthritis. Nat Rev Rheumatol. 2012 10;8(5):262-8.

[35] Sieper J. How to screen for axial spondyloarthritis in primary care? Curr Opin Rheumatol. $2012 ; 24(4): 359-62$

[36] Fautrel B, Benhamou M, Foltz V, Rincheval N, Rat A-C, Combe B et al. Early referral to the rheumatologist for early arthritis patients: evidence for suboptimal care. Results from the ESPOIR cohort. Rheumatology (Oxford). $2010 ; 49(1): 147-55$ 
Tableau 1 : Caractéristiques des 432 patients atteints de SpA axiale

\begin{tabular}{|c|c|c|c|}
\hline Variable & $\begin{array}{l}\begin{array}{l}\text { Tous les } \\
\text { patients } \\
\mathrm{N}=432\end{array} \\
\end{array}$ & $\begin{array}{l}\text { Etude } 2009 \\
\mathrm{~N}=265\end{array}$ & $\begin{array}{l}\text { Etude } 2013 \\
\mathrm{~N}=167\end{array}$ \\
\hline Sexe, masculin, N (\%) & $243(56,2)$ & $161(60,7)$ & $82(49,1)$ \\
\hline $\begin{array}{l}\text { Age au diagnostic, années, moyenne } \\
\text { (ET) }\end{array}$ & $34,2(12,5)$ & $33,1(13,1)$ & $36,0(11,3)$ \\
\hline $\begin{array}{l}\text { Age à l'apparition des premiers } \\
\text { symptômes, années, moyenne (ET) }\end{array}$ & $29,3(12,2)$ & $28,1(12,7)$ & $31,2(11,3)$ \\
\hline $\begin{array}{l}\text { Niveau d'éducation > études secondaires, } \\
N(\%)\end{array}$ & $269(62,2)$ & $167(64,5)$ & $102(61,0)$ \\
\hline Poste de fonctionnaire N/N (\%) & $142 / 269(52,8)$ & $76 / 129(58,9)$ & $66 / 140(47,1)$ \\
\hline Positivité de HLA B27 N/N (\%) & $288 / 383(75,1)$ & $191 / 242(78,9)$ & $97 / 141(68,8)$ \\
\hline BASFI (0-100), moyenne (ET) & $26,7(24,7)$ & $27,7(25,7)$ & $25,1(22,9)$ \\
\hline BASDAI (0-100), moyenne (ET) & $34,4(22,0)$ & $32,1(22,8)$ & $37,9(20,3)$ \\
\hline $\begin{array}{l}\text { Présentation clinique au cours de la } \\
\text { maladie, N/N (\%) } \\
\text { Arthrite périphérique } \\
\text { Dactylite } \\
\text { Uvéite } \\
\text { Douleurs enthésitiques } \\
\text { Maladie intestinale inflammatoire } \\
\text { Psoriasis } \\
\text { Réponse aux AINS }\end{array}$ & $\begin{array}{l}170 / 432(39,3) \\
84 / 432(19,4) \\
116 / 432(26,8) \\
121 / 374(32,3) \\
37 / 432(8,6) \\
129 / 432(29,9) \\
358 / 432(82,9)\end{array}$ & $\begin{array}{l}125 / 265(47,1) \\
130 / 265(49,0) \\
75 / 265(28,3) \\
29 / 207(14,0) \\
25 / 265(9,4) \\
79 / 265(29,8) \\
215 / 265(81,1)\end{array}$ & $\begin{array}{l}45 / 167(26,9) \\
22 / 167(13,2) \\
41 / 167(24,5) \\
92 / 167(55,1) \\
12 / 167(7,2) \\
50 / 167(29,9) \\
143 / 167 \\
(85,6)\end{array}$ \\
\hline
\end{tabular}

BASDAI : Bath Ankylosing Spondylitis Disease Activity Index,

BASFI : Bath Ankylosing Spondylitis Functional Index,

AINS : anti-inflammatoire non-stéroïdien

Les données manquantes sont $<5 \%$ excepté pour la sacro-iliite à l'IRM : 41,8\%, le BASFI : $29,7 \%$, le statut socio-économique : $37,7 \%$, les douleurs enthésitiques : $13,4 \%$, la sacro-iliite radiographique : $8,3 \%$. 
Tableau 2 : Régression linéaire univariée et multivariée visant à expliquer le délai diagnostique de la SpA axiale

\begin{tabular}{|c|c|c|c|}
\hline Variable & $\begin{array}{l}\text { Analyse } \\
\text { univariée, } \\
\text { bêta (valeur } p \text { ) }\end{array}$ & $\begin{array}{l}\text { Analyse } \\
\text { multivariée, } \\
\text { bêta (valeur p) }\end{array}$ & $\begin{array}{lr}\begin{array}{l}\text { Variable du quartile } \\
\text { inférieur } \\
\text { supérieur du } \\
\text { diagnostique }\end{array} & \begin{array}{r}\text { versus } \\
\text { délai }\end{array} \\
\end{array}$ \\
\hline Sexe : féminin & $-0,91(0,14)$ & $-0,45(0,45)$ & \\
\hline $\begin{array}{l}\text { Age plus élevé au } \\
\text { diagnostic }\end{array}$ & $0,15(<0,001)$ & $0,13(<0,001)$ & 31,4 vs 39,7 ans \\
\hline $\begin{array}{l}\text { Plus faible niveau } \\
\text { d'éducation }\end{array}$ & $-1,66(0,03)$ & $-0,61(0,44)$ & \\
\hline Année civile du diagnostic & $0,079(0,005)$ & $0,003(0,040)$ & \\
\hline $\begin{array}{l}\text { Situation professionnelle } \\
\text { (emploi rémunéré) }\end{array}$ & $-1,75(0,005)$ & $-1,12(0,070)$ & \\
\hline $\begin{array}{l}\text { Présentation clinique au } \\
\text { cours de la maladie } \\
\text {-Arthrite } \\
\text { périphérique/dactylite } \\
\text {-Douleurs enthésitiques }\end{array}$ & $\begin{array}{l}-1,92(0,016) \\
1,10(0,074)\end{array}$ & $\begin{array}{l}-1,69(0,005) \\
1,46(0,015)\end{array}$ & $\begin{array}{l}45,6 \% \text { versus } 31,3 \% \\
46,1 \% \text { versus } 55,1 \%\end{array}$ \\
\hline
\end{tabular}

Les résultats significatifs en analyse multivariée sont présentés en caractères gras. La dernière colonne représente, à titre illustratif, le niveau de chaque variable significative en analyse multivariée entre les patients du premier quartile (délai diagnostique le plus court) et ceux du dernier quartile (délai diagnostique le plus long). 